\title{
Integral Solutions of Trigonometric Knizhnik- Zamolodchikov Equations and Kac-Moody Algebras
}

By

\author{
Ivan CHEREDNIK*
}

\begin{abstract}
We use Kac-Moody algebras to get some integral solutions for trigonometric $r$-matrix KnizhnikZamolodchikov equations of type $X_{l}^{(1)}$, generalizing those in the case of Yang's $r$-matrix. where our construction gives a new interpretation and a short proof of Schechtman, Varchenko theorem.
\end{abstract}

\section{§0. Introduction}

In papers by Dotsenko, Fateev, Aomoto, Christe, Flume and forgoing papers $[1,2,3,4]$ some hypergeometric-type integral solutions were found of the Knizhnik-Zamolodchikov equation, which appeared as an equation for $n$-point correlation functions of the so-called WZW-model [5]. These correlation functions are determined in terms of primary fields (or vertex operators for Kac-Moody algebras) by means of a certain operator formalism. The concrete formulas for them (see e.g. $[6,7]$ ) are of integral type as well and should be connected with the solutions of $[1,2]$. The latter is not clarified in full (especially for arbitrary initial simple Lie algebras $\mathfrak{g}$ ).

There is another more direct interpretation of $K-Z$ equations using KacMoody algebras. It was shown in [8,9] that in some sense the so-called $\tau$-function (the coinvariant) is a generic solution of the $r$-matrix $K-Z$ equation, which is a very particular case of the universal $K-Z$ equation for arbitrary curves and vector bundles. Unlike the most general one the $r$-matrix equation can be written explicitly and does not draw in the moduli spaces of curves or bundles. It is small wonder since roughly speaking classical $r$-matrices are just in one-to-one correspondence with $\mathrm{g}$-bundles without moduli (the genus of the base curve is to be 0 or 1 in this case). By the way the proof of the above property of $\tau$ (see Theorem 1) is not far from the physical deduction of the $K-Z$ equation (see [5]).

Communicated by M. Kashiwara, April 27, 1990.

1991 Mathematics Subject Classification: 11F60, 33D70.

* Research Institute for Mathematical Sciences, Kyoto 606, Japan. On leave from A.N. Belozersky Laboratory, Bldg “A”, Moscow State University, Moscow 119899, USSR. 
The purpose of this paper is to apply $\tau$-functions to get some "natural" proof and explanation of the results from [1,2] and to generalize them to rather symmetric (perhaps non-unitary) classical $r$-matrices of type $X_{l}^{(1)}$. We follow short paper [9], where the simplest trigonometric $r$-matrix was considered and the proof was outlined with the aid of Kac-Moody algebras and the Sugawara embedding.

The author thanks V.V. Schechtman, A.N. Varchenko, T. Miwa for fruitful discussions and Research Institute for Mathematical Sciences for the kind invitation. The final version of this paper was prepared during my stay at this institute. I am grateful to A. Tsuchiya for his kind interest in this work, to M. Kashiwara for useful advice and discussions and to A. Matsuo, who helped me to improve some places of this paper, which is based on my preprint RIMS-699 with the same title.

\section{$\S 1$. The Sugawara Connection}

Let $g$ be a simple finite dimensional Lie algebra over $C$ with the Killing form $(),,\left\{g_{a}\right\}$ an orthonormal bases relative to this form. We fix a quasiunitary classical $r$-matrix, that is a function $r(\lambda)$ taking its values in $g \otimes g$, depending on $\lambda$ from some domain $0 \in U \subset C$ and satisfying the following three conditions:

$$
\begin{gathered}
r(\lambda)-t \lambda^{-1} \text { is regular for } t \stackrel{\text { def }}{=} \sum_{a} g_{a} \otimes g_{a}, \\
{\left[r^{13}\left(\lambda_{1}-\lambda_{3}\right), r^{12}\left(\lambda_{1}-\lambda_{2}\right)+r^{32}\left(\lambda_{3}-\lambda_{2}\right)\right]=\left[r^{12}\left(\lambda_{1}-\lambda_{2}\right), r^{23}\left(\lambda_{2}-\lambda_{3}\right)\right],} \\
r^{12}(\lambda)+r^{21}(-\lambda)=\Theta, \partial \Theta / \partial \lambda=0 .
\end{gathered}
$$

For $\Theta=0$ we obtain unitary nondegenerated $r$-matrices from [10]. Here and further we keep the notations

$$
\begin{gathered}
x^{1}=x \otimes 1 \otimes 1 \otimes \ldots \otimes 1, x^{2}=1 \otimes x \otimes 1 \otimes \ldots \otimes 1, \text { etc for } x \in \mathrm{g} \\
r^{i j}=\sum_{a, b} C_{a b} g_{a}^{i} g_{b}^{j}, \text { where } r=\sum_{a, b} C_{a b} g_{a} \otimes g_{b} \in \mathrm{g} \otimes \mathrm{g} .
\end{gathered}
$$

These $x^{i}, r^{i j}$ are considered to be elements of $U(\mathfrak{g}) \otimes U(g) \otimes \ldots$ for the universal enveloping algebra of $g$. We will identify elements of $g \times g \times \ldots$ def images in $U(\mathrm{~g} \times \mathrm{g} \times \ldots)=U(\mathrm{~g}) \otimes U(\mathrm{~g}) \otimes \ldots(x \times y \times z \times \ldots \stackrel{\text { def }}{=}\{x, y, z, \ldots\}$ $\left.\mapsto x^{1}+y^{2}+z^{3}+\ldots\right)$.

Given pairwise distinct $\lambda_{1}, \ldots, \lambda_{n} \in U$, we choose the local parameters $\tilde{\lambda}_{i}=$ $\lambda-\lambda_{i}$ in some (small) neighbourhoods of $\left\{\lambda_{i}\right\}$ and put formally

$$
G^{i}=\mathrm{g}\left(\left(\tilde{\lambda}_{i}\right)\right)=\left\{\sum_{k \geq p} x_{k} \tilde{\lambda}_{i}^{k}, p \in Z, x_{k} \in \mathbf{g}\right\},
$$




$$
G_{0}^{i}=\mathrm{g}\left[\left[\tilde{\lambda}_{i}\right]\right]=\left\{\sum_{k \geq 0} x_{k} \tilde{\lambda}_{i}^{k}, x_{k} \in \mathrm{g}\right\}, 1 \leq i \leq n .
$$

In fact, later on it is sufficient to assume, that $\lambda_{i}-\lambda_{j} \subset U \supset \lambda-\lambda_{i}$ for all indices, but we prefer not to divert reader's attention with this detail.

Let us introduce Lie algebras $G=\prod_{i=1}^{n} G^{i}, G_{0}=\prod_{i=1}^{n} G_{0}^{i}, \hat{G}=G \oplus C c$ with the commutator

$$
[\tilde{x}+\xi c, \tilde{y}+\zeta c]=[\tilde{x}, \tilde{y}]+c \operatorname{Res}_{\lambda}(\partial \tilde{x} / \partial \lambda, \tilde{y}) d \lambda,
$$

where $\tilde{x}=\tilde{x}(\lambda)=\prod_{i=1}^{n} x^{i}\left(\tilde{\lambda}_{i}\right), \tilde{y}=\tilde{y}(\lambda)=\prod_{i=1}^{n} y^{i}\left(\tilde{\lambda}_{i}\right) \in G,[\tilde{x}, \tilde{y}]^{i}=\left[x^{i}, y^{i}\right], \xi, \zeta \in C$,

$$
\partial \tilde{x} / \partial \lambda=\prod_{i=1}^{n}\left(\partial x^{i}\left(\tilde{\lambda}_{i}\right) / \partial \tilde{\lambda}_{i}\right) \text { and } \operatorname{Res}_{\lambda}(\tilde{x}, \tilde{y}) d \lambda=\sum_{i=1}^{n} \operatorname{Res}\left(x^{i}, y^{i}\right) d \tilde{\lambda}_{i} .
$$

The writing $\tilde{x}(\lambda)$ does not mean that $\tilde{x}$ is a function of $\lambda$, but is rather convenient.

We will consider $G^{i}$ as Lie subalgebras. . $\times 0 \times G^{i} \times 0 \times \ldots$ of $G$. The elements of $G$ are called adeles, $\hat{G}$ is the Kac-Moody one-dimensional central extension of $G$ (see [11]), $G_{0}$ and $\hat{G}_{0} \stackrel{\text { def }}{=} G_{0} \oplus C c$ are the Lie subalgebras of integer (holomorphic) adeles. To make the picture full we need principal (rational) adeles.

$$
\begin{aligned}
& \text { For } \tilde{x}=\prod_{i=1}^{n} x^{i} \in G \text { we set } G_{r}=\left\{\tilde{x}_{r}, \tilde{x} \in G\right\} \\
& \qquad \tilde{x}_{r}(\mu)=\operatorname{Res}_{\lambda}(r(\mu-\lambda), \tilde{x}(\lambda)) d \lambda \stackrel{\text { det }}{=} \sum_{i=1}^{n} \operatorname{Res}_{\lambda_{t}}\left(r\left(\mu-\lambda_{i}-\tilde{\lambda}_{i}\right), x^{i}\left(\tilde{\lambda}_{i}\right)\right) d \tilde{\lambda}_{i} .
\end{aligned}
$$

Here $\tilde{x}_{r}(\mu)$ is a $g$-valued function in $\mu \in U\left(\tilde{\mu}_{i} \stackrel{\text { def }}{=} \mu-\lambda_{i} \in U\right.$ for $\left.1 \leq i \leq n\right)$, $r(\mu-\lambda)=r\left(\mu-\lambda_{i}-\bar{\lambda}_{i}\right)$ is identified with the set of its expansions at $\lambda=\lambda_{1}, \ldots$ $\lambda=\lambda_{n}$ in terms of $\left\{\tilde{\lambda}_{i}\right\},(x \otimes y, z) \stackrel{\text { def }}{=}(y, z) x$ for $x, y, z \in g$. For any function $f(\lambda)$ taking values in $g$ let us denote by $f^{i}=f^{i}\left(\lambda_{i}\right)$ its expansion with respect to the parameter $\tilde{\lambda}_{i}$ at $\lambda=\lambda_{i}$ or (depending on the context) the image of this formal series in the $i$-th component of $U(G)=U\left(G^{1}\right) \otimes U\left(G^{2}\right) \otimes \ldots$ This agrees with the above notations $f^{1}, f^{2}$, etc for the constant $f$. Identifying (after the substitution $\mu=\lambda) \tilde{x}_{r}(\lambda)$ with $\prod_{i=1}^{n} \tilde{x}_{r}^{i}$, we will always consider $\tilde{x}_{r}$ as elements of $G$ and will include $G_{r}$ into $G$.

Properties (a) and (b) of $r$ are equivalent to

$$
\tilde{x}_{r}-\tilde{x} \in G_{0}, \quad \tilde{x}_{r}=0 \Leftrightarrow \tilde{x} \in G_{0},
$$




$$
G_{r} \text { is a Lie subalgebra of } G \text {. }
$$

This statement is from [12], where the nonunitary parametric classical YangBaxter equations were introduced. Let us prove in brief $\left(b^{\prime}\right)$ and the quasiunitary condition

$$
\operatorname{Res}_{\lambda}\left(\partial \tilde{x}_{r} / \partial \lambda, \tilde{y}_{r}\right) d \lambda=0 \text { for } \tilde{x}, \tilde{y} \in G,
$$

when $r$ satisfies $(\mathrm{a}-\mathrm{c})$. See $[13,14]$ for some details.

We put $[\tilde{x}]=\sum_{i=1}^{n}\left[x^{i}\right],\left[\sum_{k=p}^{\infty} x_{k} \tilde{\lambda}_{i}^{k}\right]=\sum_{k=p}^{-1} x_{k}\left(\lambda-\lambda_{i}\right)^{k}, x_{k} \in g$. The latter is considered as element of $G$ after the corresponding expansions at $\lambda_{1}, \ldots, \lambda_{n}$. Then $\tilde{x}_{r}=[\tilde{x}]+\tilde{x}_{0}$, where $\tilde{x}_{0}=\operatorname{Res}_{\mu}\left(r_{0}(\lambda-\mu), \tilde{x}(\mu)\right) d \mu$ for $r_{0}(\lambda)=r-\imath \lambda^{-1}$ (see (2)). The 1.h.s of (c') is equal to

$$
\begin{aligned}
& \operatorname{Res}_{\lambda}\left(\partial[\tilde{x}] / \partial \lambda+\partial \tilde{x}_{0} / \partial \lambda,[\tilde{y}]+\tilde{y}_{0}\right) d \lambda \\
& \quad=\operatorname{Res}_{\lambda}\left(\partial \tilde{x} / \partial \lambda, \tilde{y}_{0}\right) d \lambda+\operatorname{Res}_{\lambda}\left(\partial \tilde{x}_{0} / \partial \lambda, \tilde{y}\right) d \lambda,
\end{aligned}
$$

since $(\partial[\tilde{x}] / \partial \lambda,[\tilde{y}]) d \lambda$ is (the set of expansions of) a scalar rational differential form in $\lambda \in \mathbb{C} \cup \infty$ with the only possible poles at $\lambda_{1}, \ldots, \lambda_{n}$ and $\partial \tilde{x}_{0} / \partial \lambda, \tilde{y}_{0}$ are holomorphic. One has:

$$
\begin{aligned}
& \operatorname{Res}_{\lambda}\left(\partial \tilde{x} / \partial \lambda, \tilde{y}_{0}\right) d \lambda \\
& =\operatorname{Res}_{\lambda}\left(\partial \tilde{x}(\lambda) / \partial \lambda, \operatorname{Res}_{\mu}\left(r_{0}(\lambda-\mu), \tilde{y}(\mu)\right) d \mu\right) d \lambda \\
& =-\operatorname{Res}_{\lambda}\left(\tilde{x}(\lambda), \operatorname{Res}_{\mu}\left(\partial r_{0}(\lambda-\mu) / \partial \lambda, \tilde{y}(\mu)\right) d \mu\right) d \lambda \\
& =-\operatorname{Res}_{\lambda}\left(\tilde{x}(\lambda), \operatorname{Res}_{\mu}\left(\partial \check{r}_{0}(\mu-\lambda) / \partial \mu, \tilde{y}(\mu)\right) d \mu\right) d \lambda,
\end{aligned}
$$

where $\breve{r}_{0}(\lambda) \stackrel{\text { def }}{=} r_{0}^{21}(\lambda)$ (see (c)). The last term in its turn is equal to

$$
\begin{aligned}
& \operatorname{Res}_{\lambda}\left(\tilde{x}(\lambda), \operatorname{Res}_{\mu}\left(\check{r}_{0}(\mu-\lambda), \partial \tilde{y}(\mu) / \partial \mu d \mu\right) d \lambda\right. \\
& \quad=\operatorname{Res}_{\lambda} \operatorname{Res}_{\mu}\left(\left(r_{0}(\mu-\lambda), \tilde{x}(\lambda)\right), \partial \tilde{y}(\mu / \partial \mu) d \mu d \lambda\right. \\
& \quad=\operatorname{Res}_{\mu}\left(\tilde{x}_{0}(\mu, \partial \tilde{y}(\mu) / \partial \mu) d \mu=-\operatorname{Res}_{\lambda}\left(\partial \tilde{x}_{0} / \partial \lambda, \tilde{y}\right) d \lambda .\right.
\end{aligned}
$$

The latter proves $\left(\mathrm{c}^{\prime}\right)$. We have changed the order of $\operatorname{Res}_{\lambda}$ and $\operatorname{Res}_{\mu}$ in the above deductions without any comments because all the functions (series) are holomorphic.

As for $\left.b^{\prime}\right)$, we have to calculate

$$
\begin{aligned}
C & =\left[\tilde{x}_{r}, \tilde{y}_{r}\right]=\left[\operatorname{Res}_{\mu}(r(\lambda-\mu), \tilde{x}(\mu)) d \mu, \operatorname{Res}_{v}(r(\lambda-v), \tilde{y}(v)) d v\right] \\
& =\operatorname{Res}_{\mu} \operatorname{Res}_{v}\left(\left[r^{12}(\lambda-\mu), r^{13}(\lambda-v)\right], \tilde{x}(\mu) \otimes \tilde{y}(v)\right] d v d \mu,
\end{aligned}
$$

where $(x \otimes y \otimes z, a \otimes b)=(y, a)(z, b) x$. It results from (b) and from the invariance of $($,$) that$

$$
C=\operatorname{Res}_{\mu} \operatorname{Res}_{v}\left(\left[r^{23}(\mu-v), r^{12}(\lambda-\mu)\right]\right.
$$




$$
\begin{aligned}
& \left.+\left[r^{13}(\lambda-v), r^{32}(v-\mu)\right], \tilde{x}(\mu) \otimes \tilde{y}(v)\right) d v d \mu= \\
= & \operatorname{Res}_{\mu} \operatorname{Res}_{v}\left(-r^{12}(\lambda-\mu),\left[\left(r^{23}(\mu-v), \tilde{y}(v)\right), \tilde{x}(\mu)\right]\right) d v d \mu \\
& +\operatorname{Res}_{\mu} \operatorname{Res}_{v}\left(r^{13}(\lambda-v),\left[\left(r^{32}(v-\mu), \tilde{x}(\mu)\right), \tilde{y}(v)\right] d v d \mu .\right.
\end{aligned}
$$

Here $r^{12}, r^{23}, r^{13}$ should be considered as formal series in the sense of (1) (e.g. $r^{13}(\lambda-v)$ is identified with the set of the expansions of $r^{13}\left(\lambda-\bar{v}_{i}-\lambda_{i}\right)$ at the points $\left.\tilde{v}_{i} \stackrel{\text { def }}{=} v-\lambda_{i}=0,1 \leq i \leq n\right)$. The function $r^{32}(v-\mu)$ is to be replaced by the expansions of $r^{32}\left(\widetilde{v}_{i}+\lambda_{i}-\mu\right)$, which are equal to the corresponding expansions of $t^{23} \delta(v-\mu)-r^{32}\left(v-\tilde{\mu}_{i}-\mu_{i}\right)$ for the formal $\delta$-function $\delta(v-\mu)=\delta\left(\tilde{v}_{\mathrm{i}}-\tilde{\mu}_{i}\right)=$ $\sum_{k \in Z} \tilde{v}_{i}^{-1-k} \tilde{\mu}_{i}^{k}$ because of the above order of the residues. After this substitution of formal series instead of the corresponding $r$-matrices, one can interchange $\operatorname{Res}_{\mu}$ and $\operatorname{Res}_{v}$ and use (1). We arrive at the identity from [12,14]:

$$
\left[\tilde{x}_{r}, \tilde{y}_{r}\right]+[\tilde{x}, \tilde{y}]_{r}=\left[\tilde{x}_{r}, \tilde{y}\right]_{r}+\left[\tilde{x}, \tilde{y}_{r}\right]_{r}
$$

which gives $\left(b^{\prime}\right)$.

Summarizing it up we obtain from $\left(b^{\prime}, c^{\prime}\right)$ that $G_{r}$ is isotropic in the meaning of [14], i.e. appears to be a Lie subalgebra of $\hat{G}$. Condition $\left(a^{\prime}\right)$ results in

$$
\hat{G}=\hat{G}_{0} \oplus G_{r}
$$

where the sum is direct in the sense of vector spaces. Notice that $\partial G_{r} / \partial \lambda \subset G_{r}$ by virtue of (1) because $r$ depends only on the difference $\mu-\lambda$.

Given some $g$-modules $V_{1}, \ldots, V_{n}$, let us consider $V=\bigotimes_{i=1}^{n} V_{i}$ to be a $g^{n}=$ $g \times \ldots \times g$-module and hence a $G_{0}$-module under the natural projection $G_{0} \ni \tilde{x} \rightarrow \prod_{i=1}^{n} x^{i}(0)$. One has $\tilde{x} v=\sum_{i=1}^{n} x^{i}(0) v=x^{1}(0) v_{1} \otimes v_{2} \otimes \ldots \otimes v_{n}+\ldots+$ $v_{1} \otimes \ldots \otimes v_{n-1} \otimes x^{n}(0) v_{n}$ for $\tilde{x} \in G_{0}, \quad v=v_{1} \otimes \ldots \otimes v_{n} \in V$. We define the Verma module $M=M_{V}^{\sigma}$ for $\sigma \in C$ as the universal $\hat{G}$-module generated by $V$ with the above action of $G_{0}$, where $c \tilde{v}=\sigma \tilde{v}$ for $\tilde{v} \in M\left(M=\operatorname{Ind}_{\hat{G}_{0}}{ }^{\hat{\sigma}} V\right.$ if $C=\sigma$ on $V$ ).

The coinvariant (or the vector $\tau$-function) is the linear map $\pi$ taking $\tilde{v} \in M$ to the element $v \in V \subset M$ such that $\tilde{v}-v \in G_{r} M$ (see [14]). This $\pi$ depends on the choice of $G_{r}$ and hence on $r, \lambda_{1}, \ldots, \lambda_{n}$. The consistence of this defintion follows from (2).

We introduce the Sugawara elements of degree- 1 at each point $\lambda_{i}(1 \leq i \leq n)$

$$
L^{i}=\sum_{k \geq 0} \sum_{a}\left(g_{a,-1-k}^{i}\right)\left(g_{a, k}^{i}\right), x_{k}^{i} \stackrel{\text { def }}{=}\left(x \tilde{\lambda}_{i}^{k}\right)^{i}, x \in g .
$$

They belong to a completion of $U(\hat{G})$. All these $L^{i}$ are pairwise commutative. We put 


$$
R_{i}=-\sum_{j \neq i} r^{i i}\left(\lambda_{j}-\lambda_{i}\right), 1 \leq j \leq n, \rho=\sum_{a} \rho_{a} g_{a} \in U(\mathrm{~g})
$$

where $\left(t \lambda^{-1}-r(\lambda)\right)(\lambda=0)=\sum_{a} \rho_{a} \otimes g_{a}$. Given $x^{i} \in G^{i}$ (e.g. $x_{k}^{i}$ or $\left.g_{a, k}^{i}\right)$ and $1 \leq j \leq n$ one can define the elements

$$
\bar{x}^{i}=\left(x^{i}\right)_{r} \in G_{r} \text { and } x^{i, j}=\left(\bar{x}^{i}\right)^{j} \in G^{j},
$$

i.e. the $r$-extension (1) of $x^{i}=0 \times \ldots \times 0 \times x^{i} \times 0 \times \ldots \times 0$ and its $j$-component. We will use some special notations for $x \in g, k=-1$

$$
\tilde{x}^{i}=x_{-1}^{i}, \tilde{x}^{i j}=\tilde{x}^{i, j}=x_{-1}^{i, j}=\left(\left(x_{-1}^{i}\right)_{r}\right)^{j} .
$$

Let us now assume the points $\left\{\lambda_{1}, \ldots, \lambda_{n}\right\}$ to vary. To be more precise we fix some $C$-algebra $F=F_{n}$ of functions in $\lambda_{1}, \ldots, \lambda_{n}$ ensuring the inclusion $G_{r} \subset$ $G \otimes F$. The latter means that we consider $G$ as the Lie algebra with "constant" generators $\left\{g_{a, k}^{i}\right\}$ and suppose all the coefficients of series $g_{a, k}^{i, j}$ in $\widetilde{\lambda}_{i}$ to be inside $g \otimes F$. The equivalent condition is that

$$
r^{i j}\left(\lambda_{i}-\lambda_{j}\right) \in\left(g^{\otimes n}\right) \otimes F \text { for any } 1 \leq i \neq j \leq n .
$$

Later on, $\check{v} \in M \otimes F$. There is only one extension of the relations $\partial\left(g_{a, k}^{i} v\right) / \partial \lambda_{j}$ $=0$ for any indices, $v \in V$ and the natural action of $\partial / \partial \lambda_{j}$ on $F$ to the differentiation $\partial / \partial \lambda_{j}(1 \leq j \leq n)$ on $M \otimes F^{*}$

Theorem 1 [8]. a) For $\sigma^{\prime}=\sigma+1 / 2, \check{\pi}=\pi(\check{v}), 1 \leq i \leq n$

$$
\pi\left(\sigma^{\prime} \partial \check{v} / \partial \lambda_{i}+L^{i} \check{v}\right)=\left(R_{i}+\rho^{i}\right) \check{\pi}+\sigma^{\prime} \partial \check{\pi} / \partial \lambda_{i} .
$$

Corollary 1. For any $\lambda_{1}, \lambda_{2}$

$$
\left[r^{12}\left(\lambda_{1}-\lambda_{2}\right), \rho^{1}\right]+\left[\rho^{2}, r^{21}\left(\lambda_{2}-\lambda_{1}\right)\right]=\left[r^{12}\left(\lambda_{1}-\lambda_{2}\right), r^{21}\left(\lambda_{2}-\lambda_{1}\right)\right] .
$$

In particular, $\left[r^{12}\left(\lambda_{1}-\lambda_{2}\right), \rho^{1}+\rho^{2}\right]=0$ for the unitary $r($ with $\Theta=0)$.

Proof of the theorem. Setting $\check{v}=v+\sum_{s} f_{s} \check{v}_{s}$, where $f_{s} \in G_{r}, \check{v}_{s} \in M \otimes F$, $v=\pi(\check{v}) \in V \otimes F$, we will prove a) separately for $v$ and $f_{s} \check{v}_{s}$. One has

$$
L^{i} v=\sum_{a} \tilde{g}_{a}^{i} g_{a}^{i} v=\sum_{a}\left(\tilde{g}_{a}^{i i}+\rho_{a}^{i}\right) g_{a}^{i} v
$$

in notations (4). The $G_{r}$-invariance of $\pi$ gives us the formula

\footnotetext{
* For example, $\partial\left(\left(\tilde{x}^{t}\right), v\right) / \partial \lambda_{h}=\left(\partial / \partial \lambda_{k}\right)\left(\prod_{\jmath=1}^{n}\left(r\left(\tilde{\lambda}_{\jmath}+\lambda_{l}-\lambda_{l}\right), x\right)\right) v=\prod_{J=1}^{n}\left(\delta_{\jmath k} \partial / \partial \tilde{\lambda}_{h}-\delta_{l h} \partial / \partial \tilde{\lambda}_{\jmath}\right)$ $\left(r\left(\tilde{\lambda}_{J}+\lambda_{J}-\lambda_{l}\right), x\right) v$, when $v \in V$. Here $\delta_{i j}$ is the Kronecker symbol.
} 


$$
\pi\left(L^{i} v\right)=\sum_{a} \rho_{a}^{i} g_{a}^{i} v-\sum_{a, j \neq i} \tilde{g}_{a}^{i j} g_{a}^{i} v
$$

But $\sum_{a} \tilde{g}_{a}^{i j} g_{a}^{i} v=r^{j i}\left(\lambda_{j}-\lambda_{i}\right) v$ by definition. Since the derivatives on both sides of a) for $\check{v}=v$ are the same $(\pi(v)=v)$, we arrive at a).

As for $f v$ (the indices $s$ are omitted), we will use the Sugawara identity

$$
\left[L^{i}, \tilde{\mathrm{x}}\right]=-\sigma^{\prime} \partial x^{i} / \partial \lambda, \tilde{x}=\prod_{j=1}^{n} x^{j}\left(\tilde{\lambda}_{j}\right) \in G .
$$

We obtain:

$$
L^{i} f \check{v}+\sigma^{\prime} \partial(f \check{v}) / \partial \lambda_{i}=f\left(L^{i} \check{v}+\sigma^{\prime} \partial \check{v} / \partial \lambda_{i}\right)+\sigma^{\prime}\left(\partial f / \partial \lambda_{i}-\partial f^{i} / \partial \tilde{\lambda}_{i}\right) \check{v} .
$$

Formula a) will be proved if we represent the r.h.s. of the last identity as $f^{\prime} \check{w}$ for some $f^{\prime} \in G_{r}$ and $\check{w} \in M \otimes F$. It is necessary to examine its second term only. One has the decomposition $f=\sum_{j=1}^{n} \bar{f}^{j}$ (see (4a) and $r$-matrix condition (a)). Here $\bar{f}^{k}$ is a function of $\lambda-\lambda_{k}=\tilde{\lambda}_{j}+\lambda_{j}-\lambda_{k}$ because of formula (1).

Hence $\partial \bar{f}^{j} / \partial \lambda_{i}=\partial f^{j, i} / \partial \tilde{\lambda}_{i}, \quad \partial f^{i, j} / \partial \lambda_{i}=-\partial f^{i, j} / \partial \tilde{\lambda}_{j}$ for $j \neq i$ and $\partial f^{i, i} / \partial \lambda_{i}=0$ $\left(f^{i, i} \in G^{i}\right)$. We see that

$$
\begin{aligned}
\partial f / \partial \hat{\lambda}_{i}-\partial f^{i} / \partial \tilde{\lambda}_{i} & =\sum_{j \neq i}\left(\partial \bar{f}^{j} / \partial \lambda_{i}-\partial f^{j, i} / \partial \tilde{\lambda}_{i}\right)+\partial \bar{f}^{i} / \partial \lambda_{i}-\partial f^{i, i} / \partial \tilde{\lambda}_{i} \\
& =-\sum_{j \neq i} \partial f^{i, j} / \partial \tilde{\lambda}_{j}-\partial f^{i, i} / \partial \tilde{\lambda}_{i}=-\partial \bar{f}^{i} / \partial \lambda \in G_{r} .
\end{aligned}
$$

Relation (6) follows from the theorem, a) for $n=2, \check{v}=v \in V$ and the commutativity of $L^{1}$ and $L^{2}$. Really, we can choose arbitrary $v, V$.

One can find some discussion of this theorem in author's report "KacMoody algebras and Conformal Field Theory ( $r$-matrix Knizhnik-Zamolodchikov equations)", published in the Proceed of Arbeitstagung 1990.

Let us define the generalized $\boldsymbol{r}$-matrix Knizhnik-Zamolodchikov equation for $W\left(\lambda_{1}, \ldots, \lambda_{n}\right)$ taking values in $V$ and arbitrary fixed $\kappa \in C^{*}$

$$
\kappa \partial W / \partial \lambda_{i}=\hat{R}_{i} W, \hat{R}_{i}=R_{i}+\rho^{i}+\chi^{i}, 1 \leq i \leq n,
$$

where $\chi \in U(g)$ and $\left[r^{12}\left(\lambda_{1}-\lambda_{2}\right), \chi^{1}\right]+\left[\chi^{2}, r^{21}\left(\lambda_{2}-\lambda_{1}\right)\right]=0$. The last relation and identities (1) ensure the consistence of (7) for any $\kappa$ (the cross-derivative integrability conditions). The converse is true as well.

Proposition 1. Given $R_{i}=-\Sigma r^{j i}\left(\lambda_{j}-\lambda_{i}\right)(1 \leq i \neq j \leq n)$ for some function $r(\lambda)$ with the values in $g \otimes g, \rho^{\prime}=\rho+\chi \in U(g)$ assume (7) to be consistent for 
any $æ$ and $n=2$, 3. Then $r^{12}(\lambda)+r^{21}(-\lambda)=$ const, $\rho^{\prime}$ satisfies relation (6) and $r$ is a solution of functional r-matrix equation (b).

If $r(\lambda, \mu)$ is not supposed to depend on the difference $\lambda-\mu$, then the analog of Proposition 1 gives a solution of the corresponding version of (b) satisfying the relation $\partial\left(r^{12}(\lambda, \mu)\right) / \partial \lambda=\partial\left(r^{21}(\mu, \lambda)\right) / \partial \mu$, that is the general quasiunitary condition. It is possible to extend the above considerations to this case as well.

The Sugawara connection $\left\{\partial / \partial \lambda_{i} \rightarrow \sigma^{\prime} \partial / \partial \lambda_{i}+L^{i}\right\}, 1 \leq i \leq n$, is of constant type, i.e. $\partial L^{i} / \partial \lambda_{j}=0$ for any $i, j$. Hence $\tilde{W}=\exp \left(-\sum_{i=1}^{n}\left(\lambda_{i} / \sigma^{\prime}\right) L^{i}\right) \tilde{W}_{0}$ for any constant $\widetilde{W}_{0} \in M$ is its generic horisontal section. We see that $W=\pi(\widetilde{W})$ is a generic solution of (7) for $æ=-\sigma^{\prime}, \chi=0$. The problem is to give a meaning to the resulting expression for $W$ and to describe some transformation of (7) that correspond to substitutions $\widetilde{W}_{0} \rightarrow \widetilde{W}_{0}^{\prime} \in M$. The last question is connected with the next considerations.

\section{§2. The Reduction mod $\pi$ (the Basic Example)}

Later on, $\mathfrak{h} \subset g$ is a Cartan subalgebra, $\Delta\left(\Delta_{+}\right)$is the set of all (positive) roots, $\alpha_{1}, \ldots, \alpha_{l}\left(l=r k_{g}\right)$ are simple roots relative to some fixed ordering. We will choose generators $e_{\alpha} \in g_{\alpha}, f_{\alpha} \in g_{-\alpha}\left(\alpha \in \Delta_{+}\right)$in the root spaces and $h_{\alpha}=$ $\left[e_{\alpha}, f_{\alpha}\right] /\left(e_{\alpha}, f_{a}\right)$. Then $\left(h_{\alpha}, h_{\beta}\right)=\beta\left(h_{\alpha}\right)=(\alpha, \beta)$ for $\alpha, \beta \in \Delta_{+}$, where the last form on $\mathfrak{h}^{*}$ is induced by the restriction of the Killing form onto $\mathfrak{h}$. Let us denote $e_{\alpha_{p}}$, $f_{\alpha_{p}}, h_{\alpha_{p}}$ for $1 \leq p \leq l$ by $e_{p}, f_{p}, h_{p}$. We fix such a function $\eta(\alpha)= \pm 1$ on $\Delta \ni \alpha$ such that $\Delta_{+}^{\eta}=\{\alpha \in \Delta, \eta(\alpha)=1\}$ determines some other ordering on $\Delta$ (a certain element of the Weyl group of $g$ takes $\Delta_{+}$to $\left.\Delta_{+}^{\eta} ; \Delta_{-}^{\eta}=\Delta \backslash \Delta_{+}^{\eta}=-\Delta_{+}^{\eta}\right)$, $\eta(\alpha)=0$ for $\alpha \notin \Delta$.

We put for $u \in \mathbb{C}^{*}, \lambda \in \mathbb{C}, \varepsilon= \pm 1$

$$
\begin{aligned}
s_{u}(\lambda) & =\left(e^{u \lambda}-e^{-u \lambda}\right) /(2 u), c t_{u}(\lambda)=u\left(e^{u \lambda}+e^{-u \lambda}\right) /\left(e^{u \lambda}-e^{-u \lambda}\right), \\
c s_{u}^{\varepsilon}(\lambda) & =2 u e^{\varepsilon u \lambda}\left(e^{u \lambda}-e^{-u \lambda}\right)^{-1}, c s_{u}^{0}(\lambda)=0
\end{aligned}
$$

and introduce for some linear map $b: \mathfrak{h} \rightarrow \mathfrak{h}$

$$
\begin{gathered}
r(\lambda)=t c t_{u}(\lambda)+u \sum_{\alpha \in \Delta_{+}} \eta(\alpha)\left(f_{\alpha} \otimes e_{\alpha}-e_{\alpha} \otimes f_{\alpha}\right)\left(e_{\alpha}, f_{\alpha}\right)^{-1} \\
+u \sum_{p=1}^{l} b\left(h_{p}\right) \otimes h_{p}^{\prime},\left(h_{p}, h_{q}^{+}\right)=\delta_{p q} .
\end{gathered}
$$

In all the formulas below $u$ is not significant (it gives only some multipliers), but the introduction of $u$ is convenient to make clear the connection of our results and [1]. Here and further $\delta_{p q}$ is the Kronecker symbol. We will identify $b^{\prime}$ and $\mathfrak{b}^{*}$ and use the transpose $b^{*}$ of $b\left((b x, y)=\left(x, b^{*} y\right)\right.$ for $x, y \in \mathfrak{h}, \sum_{p=1}^{l} b\left(h_{p}\right) \otimes h_{p}^{\prime}$ 
$\left.=\sum_{p=1}^{l} h_{p} \otimes b^{*}\left(h_{p}^{*}\right)\right)$

Proposition 2. a) The function $r$ satisfies $r$-matrix relations (a),(b),(c) from $\S 1$ and is unitary for skew-symmetric $b$ (if $\left.b^{*}+b=0\right)$.

b) The Lie algebra $G_{r}$ consists of all rational functions $f(z)$ in $z=e^{2 u \lambda}$ with values in $\mathrm{g} \otimes \mathrm{g}$ normalized by the relations

$$
\begin{gathered}
f(z=0) \in \mathfrak{b}^{\eta}+, f(z=\infty) \in \mathfrak{b} \stackrel{\eta}{ } \\
(f(\infty))_{\mathfrak{\xi}}+(f(0))_{\mathfrak{G}}=b\left((f(\infty))_{\mathfrak{\xi}}-f(0)_{\mathfrak{\xi}}\right),
\end{gathered}
$$

where $(x)_{\mathfrak{b}}$ is the $\mathfrak{h}$-component of $x \in \mathfrak{b} \underline{\eta}$; the latter are the Borel subalgebras corresponding to $\Delta_{ \pm}^{\eta}$.

c) For the above $r$ and $\varepsilon=\eta(\alpha)$ we have

$$
\begin{aligned}
\Theta & =u \sum_{p=1}^{l}\left(b\left(h_{p}\right) \otimes h_{p}^{*}+h_{p} \otimes b\left(h_{p}^{*}\right)\right), \\
\rho & =u\left(2 \rho_{\eta}-\sum_{p=1}^{l} b\left(h_{p}\right) h_{p}^{*}\right), 2 \rho_{\eta} \stackrel{\text { def }}{=} \sum_{\alpha \in \Delta_{+}} \eta(\alpha) h_{\alpha}, \\
\tilde{f}_{\alpha}^{i j} & =c s_{u}^{\varepsilon}\left(\tilde{\lambda}_{j}+\lambda_{j}-\lambda_{i}\right) f_{\alpha}^{j}, \tilde{e}_{\alpha}^{i j}=c s_{u}^{-\varepsilon}\left(\tilde{\lambda}_{j}+\lambda_{j}-\lambda_{i}\right) e_{\alpha}^{j}, \\
\tilde{h}_{\alpha}^{i j} & =c t_{u}\left(\tilde{\lambda}_{j}+\lambda_{j}-\lambda_{i}\right) h_{\alpha}^{j}+u\left(b\left(h_{\alpha}\right)\right)^{j}(\operatorname{see}(4 \mathrm{~b})) .
\end{aligned}
$$

We will assume now that $V_{1}, \ldots, V_{n}$ from $\S 1$ are highest weight $g$-modules with the highest vectors $v_{1}, \ldots, v_{n}$ of weights $\Lambda_{1}, \ldots, \Lambda_{n}$ relative to $\mathfrak{b}_{+}$(for $\Delta_{+}$). Let us add some new points $\lambda_{n+1}, \ldots, \lambda_{n+m}(m \geq 0)$ to the old ones $\left\{\lambda_{i}\right\}$ and set $V_{n+j}=C v_{0}$, where $g$ acts in the trivial manner. Further we will use $\pi$ for $n+m$ points identifying vac $\stackrel{\text { def }}{=} v_{1} \otimes v_{2} \otimes \ldots \otimes v_{n} \in V$ with $v_{a c}=v a c \otimes v_{0} \otimes \ldots \otimes$ $v_{0} \in V^{\prime}=V \otimes\left(\bigotimes_{j=1}^{m} V_{n+j}\right)$ and $V$ with $V^{\prime}$ as well. Therefore $\pi$ will take its values in $V$. Let $\kappa \neq 0$.

We fix some $\chi \in \mathfrak{h}$ and a set $1 \leq p_{1}, p_{2}, \ldots, p_{m} \leq l$. Put $\Lambda_{n+l}=-\alpha_{p_{j}}, 1 \leq j \leq m$ (these $\Lambda_{n+j}$ have nothing to do with $V_{n+j}$ ) and

$$
\omega=\prod_{1 \leq r<s \leq n+m} s_{u}\left(\lambda_{r}-\lambda_{s}\right)^{\left(\Lambda_{1}, \Lambda_{s}\right) / \kappa} \prod_{s=1}^{n+m} \exp \left(\left(\chi+2 u \rho_{\eta}-u b^{*}(\Lambda), \Lambda_{s}\right) \lambda_{s} \kappa^{-1}\right),
$$

where $\Lambda \stackrel{\text { def }}{=} \sum_{s=1}^{n+m} \Lambda_{s}, \rho_{\eta}$ is from Proposition 2, c). Let us define the $V$-valued function in $\lambda_{1}, \ldots, \lambda_{n+m}$

$$
W=\omega \pi(\tilde{w}), \tilde{w}=\bigotimes_{i=1}^{n} v_{i} \otimes \tilde{f}_{p_{1}}^{n+1} v_{0} \otimes \ldots \otimes \tilde{f}_{p_{m}}^{n+m} v_{0} .
$$


To calculate $w \stackrel{\text { def }}{=} \pi(\tilde{w})$ one should use the $G_{r}$-invariance of $\pi$ and move $\tilde{f}_{p_{j}}^{n+j}$ from its place to other places until all the components $n+1, \ldots, n+m$ become free from $\{\tilde{f}\}$. For example, let us get rid of $\tilde{f}_{p_{1}}^{n+1}$. Here and further we will use the abbreviations

$$
j^{\prime}=p_{j}, \eta_{j}=\eta\left(\alpha_{j^{\prime}}\right) \text { for } 1 \leq j \leq m,
$$

and the formula for $1 \leq j \neq k \leq m$

$$
\left(\tilde{f}_{j^{\prime}}^{n+j, n+k}\right) \tilde{f}_{k^{\prime}}^{n+k} v_{0}=c s_{u}^{\eta_{u}}\left(\lambda_{n+k}-\lambda_{n+j}\right)\left[f_{j^{\prime}}, f_{k^{\prime}}\right]_{-1}^{n+k} v_{0}
$$

One has: $\quad \pi(\tilde{w})=-\pi\left(P\left(\operatorname{vac} \otimes v_{0} \otimes \tilde{f}_{2^{\prime}}^{n+2} v_{0} \otimes \ldots \otimes \tilde{f}_{m^{\prime}}^{n+m} v_{0}\right)\right.$,

$$
P=\sum_{i=1}^{n} c s_{u}^{\eta_{1}}\left(\lambda_{i}-\lambda_{n+1}\right) f_{1^{\prime}}^{i}+\sum_{j=2}^{m} c s_{u}^{\eta_{1}}\left(\lambda_{n+j}-\lambda_{n+1}\right)\left[f_{1^{\prime}}, f_{j^{\prime}}\right]_{-1}^{n+j}\left(\tilde{f}_{j^{\prime}}^{-1}\right)^{n+j},
$$

where $\left(f_{j^{\prime}}^{-1}\right) f_{j^{\prime}}=1$ by definition.

Next we can take away $\tilde{f}_{2^{\prime}}^{n+2}$ or $\left[f_{1^{\prime}}, f_{2^{\prime}}\right]_{-1}^{n+2}$. Now the summation will be over $i, j$ except $j=1,2$, since $g v_{0}=0$ by definition. Then one gets free the place $n+2$, can go to $n+3$ and so on. This procedure (but not the result) depends on some order of $\{1, \ldots, m\}$. Let us give the final formula.

A sequence $c=\left(j_{1}, \ldots, j_{s} ; i\right)$ of pairwise distinct indices $1 \leq j_{1}, \ldots, j_{s} \leq m$ and $1 \leq i \leq n$ will be called a chain with the origin $j_{1}$ and the end $i$. We put

$$
\tilde{\eta}_{1}=\eta\left(\alpha_{j_{1}^{\prime}}\right), \tilde{\eta}_{2}=\eta\left(\alpha_{j_{1}^{\prime}}+\alpha_{j_{2}^{\prime}}\right), \ldots, \tilde{\eta}_{s}=\eta\left(\alpha_{j_{1}^{\prime}}+\alpha_{j_{2}^{\prime}}+\ldots+\alpha_{j_{s}^{\prime}}\right) .
$$

The ordered set $d=\left\{c_{1}, \ldots, c_{r}\right\}$ is a diagram if each $1 \leq j \leq m$ belongs to some chain (or chains). Given $c$ define

$$
\begin{gathered}
C=(-1)^{s} c s_{u}^{\widetilde{\eta}_{1}}\left(\lambda_{n+j_{2}}-\lambda_{n+j_{1}}\right) c s_{u}^{\widetilde{\eta}_{2}}\left(\lambda_{n+j_{3}}-\lambda_{n+j_{2}}\right) \\
\ldots c s_{u}^{\widetilde{\eta}_{s-1}}\left(\lambda_{n+j_{s}}-\lambda_{n+j_{s-1}}\right) c s_{u}^{\widetilde{\eta}_{s}}\left(\lambda_{i}-\lambda_{n+j_{s}}\right) \\
\cdot\left[\left[\left[f_{j_{1}^{\prime}}, f_{j_{2}^{\prime}}\right], f_{j_{3}^{\prime}}\right], \ldots, f_{j_{s}^{\prime}}\right]^{i},
\end{gathered}
$$

and put $D=C_{r} C_{r-1} \ldots C_{1}$ for $d$ above.

Proposition 3. a) Fix some ordering (permutation) of $\{1, \ldots, m\}$ and consider here and below only diagrams $d$ with increasing origins of their chains (increasing diagrams). Then

$$
w=\sum_{d} D \text { vac, where } D \text { corresponds to } d .
$$

In particular $w$ and $W$ do not depend on $\sigma$.

b) Given an unordered set $y=\left\{j_{1}, \ldots, j_{q}\right\}$ of pairwise distinct $1 \leq j_{k} \leq m$ and some $1 \leq i \leq n$, we denote the sum $\sum_{d} D$ vac by $w_{i}\{y\}\left(w_{i}[y]\right)$, where $d$ runs over the 
multitude $\delta_{i}\{y\}$ (or $\delta_{i}[y]$ ) of all increasing $d$ such that $y$ belongs to (coincides with) the union of chains of $d$ with $i$ being the end. These functions do not depend on the ordering.

The proof of a) was outlined before. To prove b) let us substitute

$$
\lambda_{k}^{\prime}=\lambda_{k}+\left(\delta_{k i}+\delta_{k, n+j_{1}}+\ldots+\delta_{k, n+j_{q}}\right) \mu \text { for } \lambda_{k}
$$

where $\mu \in C, 1 \leq k \leq n+m$. Then $w_{i}[y]$ will not alter (it depends only on the differences $\lambda_{k}-\lambda_{r}$ either for $k, r \in\{i, n+y\}$ or $k, r \notin\{i, n+y\}$. But all other terms in (10) will. To make use of this observation we turn to some equivalent form of our $r$-matrix.

The element $h=\rho_{\eta}$ (Proposition 2) satisfies the defining conditions

$$
\left(h, \alpha_{p}^{\eta}\right)=1 \text { for all simple roots } \alpha_{p}^{\eta} \text { in } \Delta_{+}^{\eta} \text {. }
$$

Let us introduce the function

$$
H\left(\lambda_{1}, \ldots, \lambda_{n}\right)=\exp \left(u \sum_{i=1}^{n}\left(2 \lambda_{i} / e\right) h^{i}\right)
$$

where $e \in N$ is more or equal to the Coxeter number of $g$. One has

$$
H f_{\alpha}^{i} H^{-1}=\xi_{i}^{-(h, \alpha)} f_{\alpha}, \xi=\exp (2 u \lambda / e), \xi_{k}=\exp \left(2 u \lambda_{k} / e\right)
$$

Lemma 1. a) The function $\bar{r}^{12}\left(\lambda_{1}-\lambda_{2}\right)=H r^{12}\left(\lambda_{1}-\lambda_{2}\right) H^{-1}$ is a r-matrix; $\bar{r}(\xi \rightarrow 0)=r(z \rightarrow 0)_{\xi}=-\bar{r}(\xi \rightarrow \infty)=-r(z \rightarrow \infty)_{\mathfrak{G}}$, where $z=\xi^{e}$ (see Proposition 2$)$, $\bar{\rho}=\rho-(2 u / e) \sum_{\alpha \in \Delta_{+}} \eta(\alpha)(h, \alpha) h_{\alpha}$.

b) The element $\bar{D}=\prod_{j=1}^{m} \xi_{n+j}^{\left(h, \alpha_{J}\right)} H D H^{-1}$ has the same form as $D$ but for

$$
\overline{c s}_{u}^{\alpha}(\xi) \stackrel{\text { def }}{=} c s_{u}^{\eta(\alpha)}(\lambda) \xi^{-(h, \alpha)}=2 u \xi^{e(1+\eta(\alpha)) / 2-(h, \alpha) \eta(\alpha)}\left(\xi^{e}-1\right)^{-1}
$$

in place of $\operatorname{cs}_{u}^{\eta(\alpha)}(\lambda)$.

Obviously, $\overline{c s}_{u}^{\alpha}$ is rational in $\xi$ and tends to zero as $\xi \rightarrow 0$ or $\xi \rightarrow \infty$ for any $\alpha \in \Delta$. Let us define $\bar{w}=H w \prod_{k=1}^{n+m} \xi_{k}^{\left(h, \Lambda_{k}\right)}$ and $\bar{w}_{i}[y]$ by the same formula with $w_{i}[y]$ in place of $w$. We put

$$
\zeta=\exp (2 \mu u / e)
$$

The above substitution (11) has the form

$$
\xi_{k}^{\prime}=\xi_{k} \zeta, \xi_{k}^{\prime}=\xi_{k} \text { for } k \in\{n+y, i\}, k \notin\{n+y, i\}
$$


respectively. The function $\bar{w}_{i}[y]$ is the limit of $\bar{w}$ as $\zeta \rightarrow 0$ (or $\zeta \rightarrow \infty$ ). It proves the independence of $\bar{w}_{i}[y]$, and hence of $w_{i}\{y\}=\Sigma w_{i}\left[y^{\prime}\right], y^{\prime} \supseteq y$.

When $u \rightarrow 0$ and $r$ turns into Yang's $r$-matrix some equivalent variant of $w$ was constructed in [1] directly (the case of $s l_{2}$ was considered in [2]).

\section{§3. The Main Theorem}

We preserve the notations of $\S 2 ; \hat{R}_{i}=R_{i}+\rho^{i}+\chi^{i}$ (see (7)) are constructed for $r$ from (8). Given $1 \leq j \leq m$, omitting $\tilde{f}_{p_{1}}^{n+j}$ in (9) (or setting $\tilde{f}_{p_{j}}^{n+j}=1$ formally) one gets some function $w\{j\}$ in $\lambda_{1}, \ldots, \lambda_{n+j-1}, \lambda_{n+j+1}, \ldots, \lambda_{n+m}$. Let $W_{i}\{j\}=$ $w_{i}\{j\} \omega, W\{j\}=w\{j\} \omega$, where $\omega$ is from (9), $w_{i}\{j\}$ was defined in Proposition 3 .

Theorem 2. a) $\left(\kappa \partial / \partial \lambda_{i}-\hat{R}_{i}\right) W=-\kappa \sum_{j=1}^{m} \partial W_{i}\{j\} / \partial \lambda_{n+j}$.

$$
\text { b) } \begin{aligned}
\sum_{i=1}^{n} h_{p}^{i} W & =\sum_{k=1}^{n+m}\left(\Lambda_{k}, h_{p}\right) W, 1 \leq p \leq l, \sum_{i=1}^{n} e_{p}^{i} W \\
& =\left(e_{p}, f_{p}\right) \sum_{p_{j}=p}\left(u\left(\eta\left(\alpha_{p}\right)+b\left(h_{p}\right), \Lambda+\alpha_{p}\right)-\kappa \partial / \partial \lambda_{n+j}\right) W\{j\} .
\end{aligned}
$$

Proof. First we will reduce a) to some pure algebraic identity. One has

$$
\kappa \partial w / \partial \lambda_{i}=-\kappa \sum_{j=1}^{m} \partial w_{i}\{j\} / \partial \lambda_{n+j} .
$$

Really,

$$
\left(\partial / \partial \lambda_{n+j_{1}}+\ldots+\partial / \partial \lambda_{n+j_{s}}\right) C=\partial C / \partial \lambda_{i}
$$

for any chain $c=\left\{j_{1}, \ldots, j_{s} ; i\right\}$. Therefore $\Sigma \partial D / \partial \lambda_{n+\jmath}=\partial D / \partial \lambda_{i}$ for each diagram $d$, where we take $\partial / \partial \lambda_{n+j}$ if $d \in \delta_{i}\{j\}$ (see Proposition 3). Using (10) we prove the required formula, that makes it possible to rewrite a) in the following form.

Lemma 2. For $\omega_{k}=\kappa \partial \log \omega / \partial \lambda_{k}$

$$
=\sum_{j \neq k} c t_{u}\left(\lambda_{j}-\lambda_{k}\right)\left(\Lambda_{k}, \Lambda_{j}\right)+\left(\chi+2 u \rho_{\eta}-u b^{*}(\Lambda), \Lambda_{k}\right), 1 \leq k \leq n+m
$$

we have $\hat{R}_{i} w=\omega_{i} w+\sum_{j=1}^{m} \omega_{n+j} w_{i}\{j\}$

Proof. Let $r_{0}$ be the $r$-matrix (8) with $b=0, R_{i}^{0}=\sum r_{0}^{l k}\left(\lambda_{i}-\lambda_{k}\right)(1 \leq i \neq k \leq n)$. Then $\rho_{0}=2 u \rho_{\eta}$ and $\hat{R}_{i}-R_{i}^{0}-\rho_{0}^{i}=\chi^{i}-u \sum_{k=1}^{n} b\left(h_{p}\right)^{k}\left(h_{p}^{+}\right)^{i}$. The r.h.s. of the latter acts on each term $D$ vac from (10) as the multiplication by $\sum_{J}\left(\chi-b^{*}(\Lambda) u\right.$, 
$\Lambda_{i}-\alpha_{p}$ ), where $j$ runs over all indices such that $d \in \delta_{i}\{j\}$. This multiplier coincides with the sum $\omega_{i}-\omega_{i}^{0}+\sum_{j}\left(\omega_{n+j}-\omega_{n+j}^{0}\right)$ over the same $j$ for $\omega_{k}^{0}$ which are defined for $r_{0}$ and $\chi=0$. Hence it is sufficient to prove the lemma setting $r=r_{0}, \chi=0$.

We remind that multitudes $\delta_{i}\{y\}, \delta_{i}[y]$ from Proposition 3 depended on some fixed ordering on $\{1, \ldots, m\}$. For any $1 \leq j \leq m$ we can change the ordering transposing $j$ and the maximal element among $\{1, \ldots, m\}$. Let us denote by $\delta_{i}^{j}\{y\}, \delta_{i}^{i}[y]$ the corresponding multitudes. The main idea of the proof is to use Theorem 1 for $\sigma^{\prime}=\sigma+1 / 2=0$ (owing to Proposition $3 w$ does not depend on $\sigma$ ). It is convenient to set $i=n$ (all $i$ are on equal grounds).

We have

$$
\begin{aligned}
\left(R_{n}+\rho^{n}\right) w & =\pi\left(v_{1} \otimes \ldots \otimes v_{n-1} \otimes L^{n} v_{n} \otimes \tilde{f}_{1^{\prime}}^{n+1} v_{0} \otimes \ldots \otimes \tilde{f}_{m^{\prime}}^{n+m} v_{0}\right) \\
& =\omega_{n} w+\sum_{\alpha \in \Delta_{+}} \pi\left(\tilde{e}_{\alpha}^{n n} f_{\alpha}^{n} \tilde{w}\right) /\left(e_{\alpha}, f_{\alpha}\right),
\end{aligned}
$$

where $\tilde{w}$ is from (9). We omitted the terms $\tilde{f}_{\alpha}^{n} e_{\alpha}^{n}$ of $L^{n}$, since $e_{\alpha}^{n} v_{n}=0$ by definition. Using the $G_{r}$-invariance of $\pi$ we will remove $\tilde{e}_{\alpha}^{n n}$ from its place to other components. There is no need to move $\tilde{e}_{\alpha}^{n n n}$ to the left, since $\tilde{e}_{\alpha}^{n i}$ is proportional to $e_{\alpha}^{i}$ and annihilates $v_{i}$ (see Proposition 2).

To calculate the contribution of $\tilde{e}_{\alpha}^{n}$ to the $(n+k)$-th component for $1 \leq k \leq m$ one can use the formula

$$
\begin{aligned}
\tilde{e}_{a}^{n, n+k} f_{k^{\prime}}^{n+k} v_{0} & =\left[\tilde{e}_{\alpha}^{n, n+k}, \tilde{f}_{k^{\prime}}^{n+k}\right] v_{0} \\
& =c s_{u}^{-\eta(\alpha)}\left(\lambda_{n+k}-\lambda_{n}\right)\left(\left[e_{\alpha}, f_{k^{\prime}}\right]_{-1}^{n+k}+\text { const. } v_{0}\right) .
\end{aligned}
$$

Here the term const. $v_{0}$ emerges due to the action of the central element $c$ of $\hat{G}$ and is not equal to zero only as $\left(e_{\alpha}, f_{k^{\prime}}\right) \neq 0$. In this case $\alpha=\alpha_{k^{\prime}}$ and $\left[e_{a}, f_{k^{\prime}}\right]=$ $h_{k^{\prime}}\left(e_{k^{\prime}}, f_{k^{\prime}}\right)$. If $\left(e_{\alpha}, f_{k^{\prime}}\right)=0$ then (12) is zero or propositional to $\tilde{e}_{\bar{a}}^{n+k} v_{0}$ for $\tilde{\alpha}=$ $\alpha-\alpha_{k^{\prime}}\left(\tilde{\alpha}\right.$ has to be from $\Delta_{+}$, since $\alpha_{k^{\prime}}$ is a simple root, $\left.\widetilde{\alpha} \neq 0\right)$. We remind that $k^{\prime}=p_{k}$ for $1 \leq k \leq m$.

The next step is to take away $\tilde{e}_{\bar{a}}^{n+k}$ from its component. We use (12) for $n+k, n+\tilde{k}, \tilde{\alpha}$ instead of $n, n+k, \alpha$, where $0 \leq \tilde{k} \neq k$. Note that at the second and further steps $\tilde{e}_{\tilde{a}}^{n+k}$ can go to the $n$-th component, since we have already got the term $f_{a}^{n} v_{n}$ there (after the first step). If $\tilde{k}=0$ then we obtain $\left[\tilde{e}_{\tilde{\alpha}}^{n+k, n}, f_{\alpha}^{n}\right] v_{n}$ after the second step that is proportional to $\left[e_{\alpha}, f_{\alpha}\right]^{n} v_{n}$. The chain of transformations will end at that. We stop it as well if $\tilde{k} \geq 1$ and $\tilde{\alpha}=\alpha_{\tilde{k}^{\prime}}$.

Thus we obtain the following three cases:

a) the above successive transformations are finished at some place $n+j>n$ and we keep only the term $\left[e_{j^{\prime}}, f_{j^{\prime}}\right]_{-1}^{n+j} v_{0}=\left(e_{j^{\prime}}, f_{j^{\prime}}\right) \tilde{h}_{j^{\prime}}^{n+j} v_{0}$ from the last commutator (12); 
$\left.\mathrm{a}^{\prime}\right)$ it is stopped at the same place but we take the other term const. $v_{0}$;

b) it is over at the $n$-th place.

The result of the whole procedure is the sum of the terms of type $v=a, a^{\prime}, b$, that are in one-to-one correspondence with chains $c=\left(j_{1}, \ldots, j_{s} ; n\right)$. Each term can be written as follows.

For $y$ being the support of $C$ (i.e. the unordered set $\left.\left\{j_{1}, \ldots, j_{s}\right\}\right)$ we introduce $\tilde{w}\{y\}$ and $w\{y\}$ by formula (9), where $\tilde{f}_{p_{j}}^{n+j}$ are omitted for $j \in y$ (cf. the definition of $w\{j\})$. Put

$$
\begin{aligned}
\varphi_{c} & =(-1)^{s} c_{u}^{-\widetilde{\eta}_{s}}\left(\lambda_{n+j_{s}}-\lambda_{n}\right) \ldots c s_{u}^{-\widetilde{\eta}_{1}}\left(\lambda_{n+j_{1}}-\lambda_{n+j_{2}}\right) \\
& =c s_{u}^{\widetilde{\eta}_{1}}\left(\lambda_{n+j_{2}}-\lambda_{n+j_{1}}\right) c s_{u}^{\widetilde{\eta}_{2}}\left(\lambda_{n+j_{3}}-\lambda_{n+j_{2}}\right) \ldots c s_{u}^{\widetilde{\eta}_{s}}\left(\lambda_{n}-\lambda_{n+j_{s}}\right), \\
f_{c} & =\left[\left[\left[f_{j_{1}^{\prime}}, f_{j_{2}^{\prime}}\right], f_{j_{3}^{\prime}}\right], \ldots, f_{j_{s}^{\prime}}\right] \in g_{-\alpha_{c}}, \alpha_{c}=\alpha_{j_{1}^{\prime}}+\ldots+\alpha_{j_{s}^{\prime}} .
\end{aligned}
$$

We suppose that $\alpha_{c}=\alpha$ in cases a), $\mathrm{a}^{\prime}$ ) and set $C^{a^{\prime}}=\varphi_{c}$,

$$
C^{a}=\varphi_{c}\left(e_{\alpha}, f_{c}\right)^{-1}\left[\left[\left[e_{\alpha}, f_{j_{s}^{\prime}}\right], f_{j_{s-1}^{\prime}}\right], \ldots, f_{j_{1}^{\prime}}\right]_{-1}^{n+j_{1}}=(-1)^{s-1} \varphi_{c} \tilde{h}_{j_{1}^{\prime}}^{n+j_{1}}
$$

Here the last equality follows from the identity

$$
\begin{aligned}
\left(f_{c}, e_{\alpha}\right)\left(\alpha_{j_{1}^{\prime}}, \alpha_{j_{1}^{\prime}}\right) & =\left(\left[\left[\left[f_{j_{1}^{\prime}}, h_{j_{1}^{\prime}}\right], f_{j_{2}^{\prime}}\right], \ldots, f_{j_{s}^{\prime}}\right], e_{\alpha}\right) \\
& =(-1)^{s-1}\left(h_{j_{1}^{\prime}},\left[\left[\left[e_{\alpha}, f_{j_{s}^{\prime}}\right], f_{j_{s-1}^{\prime}}\right], \ldots, f_{j_{1}^{\prime}}\right]\right) .
\end{aligned}
$$

Let $C^{b}=\varphi_{c}^{\prime} c s_{u}^{-\eta\left(\alpha-\alpha_{c}\right)}\left(\lambda_{n}-\lambda_{n+j_{1}}\right) e_{\alpha-\alpha_{c}}^{n}$ for $\alpha-\alpha_{c} \in \Delta_{+}$, where $\varphi_{c}^{\prime}$ is defined like $\varphi_{c}$ but for

$$
\tilde{\eta}_{s}^{\prime}=\eta(\alpha), \tilde{\eta}_{s-1}^{\prime}=\eta\left(\alpha-\alpha_{j_{s}^{\prime}}\right), \tilde{\eta}_{s-2}^{\prime}=\eta\left(\alpha-\alpha_{j_{s}^{\prime}}-\alpha_{j_{s-1}^{\prime}}\right), \ldots
$$

Elsewhere $C^{v}=0$.

Thus, when reducing $\tilde{e}_{\alpha}^{n}$ by the above procedure we obtain the sum over all $c$ of the terms $\left(C^{a}+\right.$ const. $C^{a^{\prime}}+$ const. $\left.C^{b}\right)\left(f_{c}^{n} \tilde{w}\{y\}\right)$. The constants of $C^{a^{\prime}}$ and $C^{b}$ will not be significant later. Next we can get rid of the rest $\tilde{f}_{k^{\prime}}^{n+k}(1 \leq k \leq m$, $k \notin y$ ) by means of the construction of $\S 2$. We do it with respect to the initial ordering upon the restriction to $\{1, \ldots, m\} \backslash y$.

Let us take $\pi$ of the above sum and change $-\tilde{h}_{j_{1}^{\prime}}^{n+j_{1}}$ in $C^{a}$ by

$$
\tilde{\omega}_{n+j_{1}}=\sum_{k=1}^{n+m} c t_{u}\left(\lambda_{k}-\lambda_{n+j_{1}}\right)\left(\Lambda_{k}, \Lambda_{n+j_{1}}\right)+c t_{u}\left(\lambda_{n}-\lambda_{n+j_{1}}\right)\left(\Lambda_{n}-\alpha, \Lambda_{n+j_{1}}\right),
$$

where $k \neq n, k \notin n+y$ (we use the $G_{r}$-invariance of $\pi$ ). By repeating the procedure of $\S 2$ one gets the formula

$$
\left(R_{n}+\rho^{n}-\omega_{n}\right) w=\sum_{j=1}^{m} \sum_{d}\left(D^{a}+D^{a^{\prime}}+D^{b}\right)\left(f_{c_{1}}^{n} \mathrm{vac}\right),
$$

where $d=\left(c_{1}, \ldots, c_{r}\right)$ runs over all diagrams from $\delta_{n}^{j}\{j\}, D^{v}=C_{r} \ldots C_{2} C_{1}^{v}$, the 
multitudes $\delta_{n}^{j}\{y\}, \delta_{n}^{j}[y]$ for any $y$ were defined above. Combining formulas (13) for $\varphi_{c}, f_{c}, C^{a}$ we see that $D^{a}\left(f_{c_{1}}^{n} \mathrm{vac}\right)$ is proportional to $D$ vac from $(10)$, where the multiplier is equal to $\widetilde{\omega}_{j_{1}+n}$. This observation is the central point in our proof of the lemma.

Finally, one comes to the identity

$$
R_{n} w+\rho^{n} w-\omega_{n} w=\sum_{y} \sum_{j \in y}\left(\omega_{n+j} S_{y}^{j}+Q_{y}^{j}\right), y \subset\{1, \ldots, m\}
$$

where $S_{y}^{j}=\sum_{d} D$ vac, $d \in \delta_{n}^{j}[y]$; each $Q_{y}^{j}$ depends either on the differences $\lambda_{k}-\lambda_{t}$ for $k, t \in\{n, n+y\}$ or on those for $k, t \notin\{n, n+y\}$ and corresponds to some contribution of $D^{a^{\prime}}, D^{b}, \omega_{n+j}-\tilde{\omega}_{n+j}$. The sum for $S_{y}^{j}$ does not depend on $j$ (it does not depend on the initial ordering because of Proposition 3, b)). Therefore

$$
S_{y}^{j}=w_{n}[y] \text { and } \sum_{y} \sum_{j \in y} \omega_{n+j} S_{y}^{j}=\sum_{y}\left(\sum_{j \in y} \omega_{n+j}\right) w_{n}[y]=\sum_{j=1}^{m} \omega_{n+j} w_{n}\{j\} .
$$

The latter coincides with the r.h.s. of the required identity and the only thing left is to prove that the remainder $Q=\sum_{y} \sum_{j \in y} Q_{y}^{j}$ equals zero identically. We will use the same trick as when proving Proposition 3.

Given $y=\left\{j_{1}, \ldots, j_{q}\right\}$ let us make substitution (11) for $i=n$ and use the conjugation of Lemma 1 (i.e. replace $r, D, w_{n}[y]$ by $\bar{r}, \bar{D}, \bar{w}_{n}[y]$ etc). It was shown that $\bar{w}_{n}[y]$ does not depend on $\zeta$ and $\bar{w} \rightarrow \bar{w}_{n}[y]$ as $\zeta \rightarrow 0$ or $\zeta \rightarrow \infty$. By virtue of Lemma 1 , a) the corresponding limits for $\bar{R}_{n}$ are some constants with the sum equal to zero. The latter holds good for

$$
\begin{aligned}
& \lim _{\zeta \rightarrow 0, \infty}\left(\bar{R}_{n} \bar{w}+\rho^{n} \bar{w}-\omega_{n} \bar{w}-\sum_{j=1}^{m} \omega_{n+j} \bar{w}_{n}\{j\}\right) \\
& \quad=\lim _{\zeta \rightarrow 0, \infty}\left(\bar{R}_{n}\right) \bar{w}_{n}[y]+\lim _{\zeta \rightarrow 0, \infty}\left\{\left(\rho, \Lambda_{n}\right)-\omega_{n}+\sum_{j=1}^{m}\left(\left(\rho, \Lambda_{n+j}\right)-\omega_{n+j}\right)\right\} \bar{w}_{n}[y],
\end{aligned}
$$

because $\omega_{k}(\zeta=0)+\omega_{k}(\zeta=\infty)=2\left(\rho, \Lambda_{k}\right)$ for every $k$. Here we have used the following formula:

$$
\rho^{i} w=\left(\rho, \Lambda_{i}\right) w+\sum_{j=1}^{m}\left(\rho, \Lambda_{n+j}\right) w_{i}\{j\}, 1 \leq i \leq n .
$$

Every term of $Q_{y^{\prime}}^{j}$ is either from the sum $\sum_{d} D^{b}\left(f_{c_{1}}^{n}\right.$ vac $)$ over $d \in \delta_{n}^{j}\left[y^{\prime}\right]$ or has the form $\beta w_{n}\left[y^{\prime}\right]$ where $\beta$ is a linear combination of functions $1, c t_{u}\left(\lambda_{k}-\right.$ $\left.\lambda_{n+j}\right), k \neq n+j, k \in\left\{n, n+y^{\prime}\right\}$. In any case $\bar{Q}_{y^{\prime}}^{j} \rightarrow 0$ as $\zeta \rightarrow 0, \infty$ if $y^{\prime} \neq y$, since it is so for $\bar{w}_{n}\left[y^{\prime}\right]$ (see the proof of Proposition 3). Thus $\bar{Q}(\zeta \rightarrow 0, \infty)=\sum_{j \in y} \bar{Q}_{y}^{j}$, where $\bar{Q}_{y}^{j}$ does not depend on $\zeta$. One has: $\lim _{\zeta \rightarrow 0} \bar{Q}+\lim _{\zeta \rightarrow \infty} \bar{Q}=2 \sum_{j \in y} \bar{Q}_{y}^{j}$. However, it 
was checked that this sum is to be equal to zero. Hence $\sum_{j \in y} Q_{y}^{j}=0$ for any $y$ and $Q=0$.

The first of the two statements b) can be easily verified directly owing to the proportionality of $\tilde{f}_{p_{j}}^{j k}$ to $f_{p_{j}}^{k}$ for any indices (see Proposition 2 and formula (10)). It is not difficult to check the other by writing everything out. We will use the way that is close to the proof of Lemma 2. Given $p$ set $\varepsilon=\eta\left(\alpha_{p}\right)$.

Let us choose the infinite point $\lambda_{\infty}$ such that $z=e^{2 u \hat{\lambda}}$ is equal to 0 or $\infty$ for $\varepsilon=1, \varepsilon=-1$ respectively $\left(z^{\varepsilon}\left(\lambda_{\infty}\right)=0\right)$. We add $\lambda_{\infty}$ to $\left\{\lambda_{n+1}, \ldots, \lambda_{n+m}\right\}$ and put

$$
G^{\infty}=\mathbf{g}\left(\left(z^{\varepsilon}\right)\right), G_{0}^{\infty}=z^{\varepsilon} \mathfrak{g}\left[\left[z^{\varepsilon}\right]\right] \oplus \mathfrak{b}_{+}^{\eta} .
$$

One must extend the old $G_{r}$ to the new one by adjoining $\mathfrak{g}\left[\left[z^{-\varepsilon}\right]\right] \oplus \mathfrak{n} \underline{\eta}$ ("rational" functions with the only pole at $\infty$ ), where $\mathfrak{n}_{ \pm}^{\eta}=\left[\mathfrak{b}_{ \pm}, \mathfrak{b}_{ \pm}\right]$is the radical of the Borel subalgebra $\mathfrak{b}_{ \pm}$. Starting with the free $\mathfrak{b}_{ \pm}^{\eta}$-module $\mathrm{V}_{\infty}=U\left(\mathfrak{b}_{+}^{\eta}\right) v_{0}$ we set $\tilde{f}_{p,}^{\infty} \stackrel{\text { def }}{=} e_{p}^{\infty}$ and $\Lambda_{\infty}=0$ by definition.

Note that the value of a rational function $x(\lambda) \in G_{r}$ or an adele $\tilde{x}^{\prime} \in G \times G_{0}^{\infty}$ at $\lambda_{\infty}$ belongs to the Lie algebra $\mathfrak{g}_{\infty}=G_{0}^{\infty} / z^{\varepsilon} G_{0}^{\infty}=\mathfrak{b}_{+}^{\eta} \oplus z^{\varepsilon} \mathfrak{n}-\bmod z^{\varepsilon}$. Let us consider $V_{\infty}$ as $G_{0}^{\infty}$-module with respect to the projection $G_{0}^{\infty} \rightarrow \mathfrak{b}_{+}^{\eta}$ and define $\pi^{\prime}$ for $\left\{\lambda_{1}, \ldots, \lambda_{n+m}, \lambda_{\infty}\right\}$ taking values in $V \otimes V_{\infty}$ modulo the diagonal action of $n_{+}^{\eta}$, i.e. in $V \otimes v_{0} \cong V$. As a mater of fact $z^{\varepsilon}=0$ is a point of bad reduction for the bundle of Lie algebras, connected with $r$, since $g_{\infty}$ is not semisimple (see $[13,14]$ for details). But all the above constructions hold good and we get functions $\tilde{w}^{\prime}$, $w^{\prime}$ and $W^{\prime}$ (for the same $\omega$ ).

Let us verify that $w^{\prime}=-\sum_{i=1}^{n} e_{p}^{i} w$. In accordance with the procedure of $\S 2$ we cancel $\tilde{f}_{p_{1}}^{n+1}, \ldots, \tilde{f}_{p_{m}}^{n+m}$ from

$$
w^{\prime}=\pi^{\prime}\left(\operatorname{vac} \otimes \tilde{f}_{p_{1}}^{n+1} v_{0} \otimes \ldots \otimes \tilde{f}_{p_{m}}^{n+m} v_{0} \otimes e_{p}^{\infty} v_{0}\right)
$$

using the invariance of $\pi^{\prime}$. It is unnecessary to move any $\tilde{f}_{\alpha}^{n+j}$ to the infinite place, since $\left[\tilde{f}_{\alpha}^{n+j, \infty}, e_{p}^{\infty}\right] \in z^{\varepsilon} g\left[\left[z^{\varepsilon}\right]\right]$ acts trivially on $V_{\infty}$. Hence $w^{\prime}=\pi^{\prime}(w \otimes$ $\left.e_{p}^{\infty} v_{0}\right)=-\sum_{i=1}^{n} e_{p}^{i} w$. On the other hand we can first get rid of $e_{p}^{\infty}$ by means of the same invariance. The contribution to the components $1, \ldots, n$ and those with indices $\left\{n+j, p_{j} \neq p\right\}$ equals zero $\left(\mathfrak{n}_{+} v_{i}=0\right.$ for $1 \leq i \leq n,\left[e_{p}, f_{q}\right]=0$ for $\left.p \neq q\right)$. As for $p_{j}=p$, one has $e_{p}^{\infty, n+J}=-e_{p}$ and

$$
e_{p} \tilde{f}_{p_{j}}^{n+j} v_{0}=\left[e_{p}, \tilde{f}_{p}^{n+j}\right] v_{0}=\left(e_{p}, f_{p}\right) \tilde{h}_{p}^{n+j} v_{0}
$$

Next we substitute $\tilde{h}_{p}^{n+j, \infty}+\sum_{k \neq n+j} \tilde{h}_{p}^{n+j, k}$ for $-\tilde{h}_{p}^{n+j}$ and replace the last sum by $-\kappa \partial \log \omega / \partial \lambda_{n+j}$. The action of $\widetilde{h}_{p}^{n+j, \infty}=u\left(\varepsilon h_{p}+b\left(h_{p}\right)\right)^{\infty}$ on $\tilde{w}\{j\} \otimes v_{0}$ coincides modulo $\pi^{\prime}$ with the action of $-u \sum_{k=1}^{n+m}\left(\varepsilon h_{p}+b\left(h_{p}\right)\right)^{k}$ on $\tilde{w}\{j\}$ and is the multi- 
plication by $u\left(\varepsilon h_{p}+b\left(h_{p}\right), \Lambda+\alpha_{p}\right)$. Hence we arrive at the second formula from b). By the way the first one can be proved in the same way as well.

The theorem makes it possible to get solutions of Knizhnik-Zamolodchikov equation (7) for the above trigonometric $\hat{R}_{i}$. Let $F_{n} \subset F_{n+m}$ be a pair of $C$ algebras of functions in $\lambda_{1}, \ldots, \lambda_{n}$ and in $\lambda_{1}, \ldots, \lambda_{n+m}$ respectively satisfying condition (5). We suppose the existence of some map int from $V \otimes F_{n+m} \omega$ to a certain $F_{n}$-algebra of functions in $\lambda_{1}, \ldots \lambda_{n}$ taking values in $V$ with the following properties. It should be linear under the action of End $V \otimes F_{n}$, commute with $\partial / \partial \lambda_{i}(1 \leq i \leq n)$ and vanish on the derivatives $\partial w_{i}\{j\} / \partial \lambda_{n+j}(1 \leq j \leq m)$.

Roughly speaking int is an integral $\int \ldots \int(\cdot) \omega d \lambda_{n+1} \ldots d \lambda_{n+m}$ over some relatively compact $\boldsymbol{C}$-chain in $\boldsymbol{C}^{m}=\left\{\left(\lambda_{n+1}, \ldots, \lambda_{n+m}\right)\right\}$ with the boundary on the affine hyperplanes $\left\{c t_{u}\left(\lambda_{k}-\lambda_{s}\right)=0,1 \leq k \neq s \leq n+m\right\}$ depending on $\lambda_{1}, \ldots, \lambda_{n}$. This integral can be viewed algebraically as a multi-dimensional residue. By all means it results from Theorem 2 that given int the function $\operatorname{int}(W)$ is to be a solution of (7). A more detailed discussion of this construction requires some analysis beyond the framework of this paper (see e.g. [3]).

Concluding remarks. a) In the degenerated case $u \rightarrow 0$ the above formulas coincide with the integral formulas from [1]. But this passage does not exhaust all the connections between the trigonometric and Yang's $K-Z$ equations. If one uses the variables $z_{i}=\exp \left(2 u \lambda_{i}\right)$ the resulting equation will be close to the Yang one, but will have extra singularities (poles) at the hyperplanes $\left\{z_{i}=0\right\}$. The appearence of these poles has direct reference to the affine character of the trigonometric $r$-matrix equations (see [15]). Varchenko found some mapping from Yang's $(n+1)$-point equation to the trigonometric $n$-point one. This observation makes it possible to apply the above trigonometric construction to clarify some properties of the integral formulas for the usual $K-Z$ equation.

b) In $\S 2,3$ we have been considering the $r$-matrices that are nonunitary generalizations of $r$-matrices from [10] of type $X_{l}^{(1)}$ and with empty $\Gamma_{1}, \Gamma_{2}$ (see Section 6.4). I hope that there is some straightforward version of the above construction for general $\Gamma_{1}, \Gamma_{2}$. As for $X_{l}^{(s)}, s \neq 1$, there are some hopes as well. But formula (9) should be of more complicates nature.

c) The natural generalization of Theorem 2 is as follows. One can consider the algebra $\mathrm{g}(A)$ from [11] for some symmetrizable matrix $A$ and use the invariant form and the decomposition of Theorem 2.2 to define $t \in \mathfrak{g}(A) \otimes \mathrm{g}(A)$ and trigonometric $r$-matrices like those from Proposition 2. The $r$-matrix KniznikZamolodchikov equation, the coinvariant $\pi$ and formula (10) for $w$ are meaningful for any $A$ (cf.[1]). There are some difficulties when extending the proof of Theorem 2 to the case of arbitrary $A$ (the Lie algebra $\mathrm{g}(A)$ can be infinite dimensional and one has to be precise with the definition of $L^{i}$ and the above calculations). However this theorem is valid in general and can be deduced from 
the particular case considered in $\S 3$. The idea is as follows. We must check that the identity from Lemma 2 does not draw in the Chevalley-Serre relations $\left(\operatorname{ade}_{\mathrm{i}}\right)^{1-a_{l}} e_{j}=0=\left(\operatorname{ad} f_{i}\right)^{1-a_{l l}} f_{j}$ (see [11], §3.3). It can be established, since $\Lambda_{1}, \ldots, \Lambda_{k}, f_{p_{1}}, \ldots, f_{p_{m}}$ and $l=r k_{g}$ are indeterminate. This means that we can assume $\left(\Lambda_{k}, \Lambda_{r}\right), 1 \leq k, r \leq n+m$, to be independent variables and Lemma 2 is separated into several identities. Together they give the generalization of Theorem 3 for any $A$. I hope one will manage to prove it without this reasoning with the aid of the method of $\S 3$.

d) One can consider the elements of the Virasoro algebra of any degree relative to the Sugawara embedding. Moreover there is a definition of analogical elements for any initial Casimir operators of $g$ instead of the quadratic one (due to Malikov and Hayashi). It is not difficult to calculate the action of these elements on $v \in V$ modulo $\pi$. For $\sigma^{\prime}=0$ one gets a family of pairwise commuting functions in $\lambda_{1}, \ldots, \lambda_{n}$ with their values in End $V, V=V_{1} \otimes \ldots \otimes V_{n}$. It is interesting to find their common eigenvectors by means of some Bethe equations or any other methods.

\section{References}

[1] Schechtman V.V. and Varchenko A.N., Integral representations of N-point conformal correlations in the WZW model, Preprint Max-Planck-Institute MPI/89-51, Bonn (1989).

[2] Date E., Jimbo M., Matsuo A. and Miwa T., Hypergeometric-type integrals and the $S L_{2}(C)$ Knizhnik-Zamolodchikov equation, Preprint RIMS 667, Kyoto (1989).

[3] Schechtman V.V. and Varchenko A.N., Arrangements of hyperplanes and Lie algebra homology, Invent. Math., (1991).

[4] Matsuo A., An application of Aomoto-Gelfand hypergeometric functions to the $S U(n)$ Knizhnik-Zamolodchikov equations, Preprint RIMS 683, Kyoto (1990).

[5] Knizhnik V.G. and Zamolodchikov A.B., Current algebra and Wess-Zumino models in two dimensions, Nucl. Phys. B, 247 (1984), 83-103.

[6] Gerasimov A., Marshakov A., Morosov A., Olshanetsky M. and Shatashvili S., Wess-Zumino model as a theory of free fields, Preprint Moscow (1989).

[7] Feigin B. and Frenkel E., Representations of affine Kac-Moody algebras and bosonization, V.G. Knizhnik memorial volume, World Scientific, Singapore, 1989.

[8] Cherednik I.V., Generalized braid groups and local $r$-matrix systems, Doklady Akad. Nauk SSSR, 307 (1989), 27-34.

[9] On solutions of the trigonometric Knizhnik-Zamolodchikov equation, to appear in Funct. Anal Appl., (1991).

[10] Belavin A.A. and Drinfeld V.G., On solutions of the classical Yang-Baxter equation for simple Lie algebras, Funct. Anal Appl., 16 (1982), 1-29.

[11] Kac V., Infinite dimensional Lie algebras, Birkhäuser, Boston, 1983.

[12] Cherednik I.V., On a definition of $\tau$-functions for generalized affine Lie algebras, Funct. Anal. Appl., 17 (1983), 93-95.

[13] _ Algebraic methods in soliton theory, to be published by World Sci., 1992.

[14] Functional realizations of basic representations of factorisable groups and Lie algebras, Funct. Anal. Appl., 19 (1985), 36-52.

[15] Monodromy representations for generalized Knizhnik-Zamolodchikov equations and Hecke algebras, Preprint ITF-89-74E, Kiev (1989), to appear in Publ. of RIMS. 